\title{
Findings from post-intervention analysis of pre-eclampsia/ eclampsia in Ebonyi State
}

Salisu Mohammed Ishaku

Population Council

Follow this and additional works at: https://knowledgecommons.popcouncil.org/departments_sbsr-rh

Part of the Demography, Population, and Ecology Commons, Family, Life Course, and Society Commons, International Public Health Commons, Maternal and Child Health Commons, and the Women's Health Commons How does access to this work benefit you? Let us know!

\section{Recommended Citation}

Ishaku, Salisu Mohammed. 2018. "Findings from post-intervention analysis of pre-eclampsia/eclampsia in Ebonyi State," Ending Eclampsia Research Brief. Abuja: Population Council. 
Findings from post-

intervention analysis of

pre-eclampsia/

eclampsia-in Ebonyi State
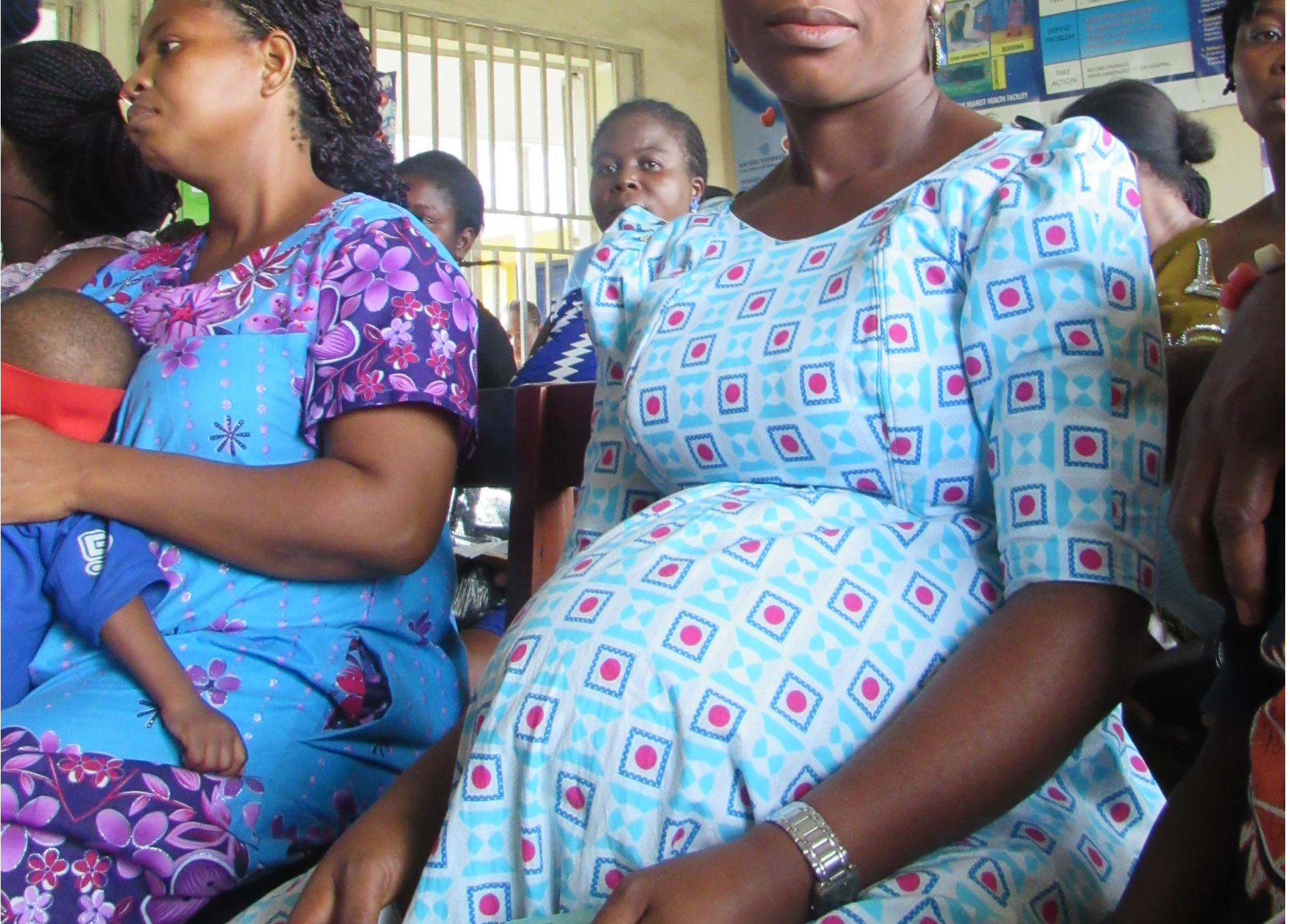

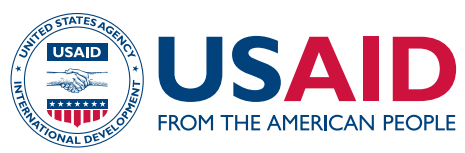

Made possible by the generous support of the American people through the United States Agency

for International Development (USAID).
The Population Council conducts research and delivers solutions that improve lives around the world. Big ideas supported by evidence: It's our model for global change. popcouncil.org

(c) 2018 The Population Council, Inc.
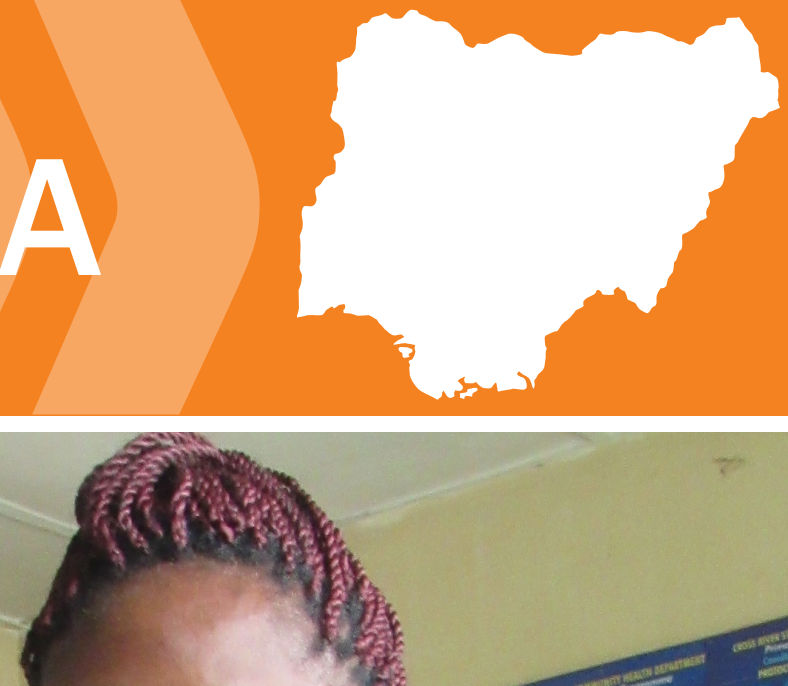

(1)
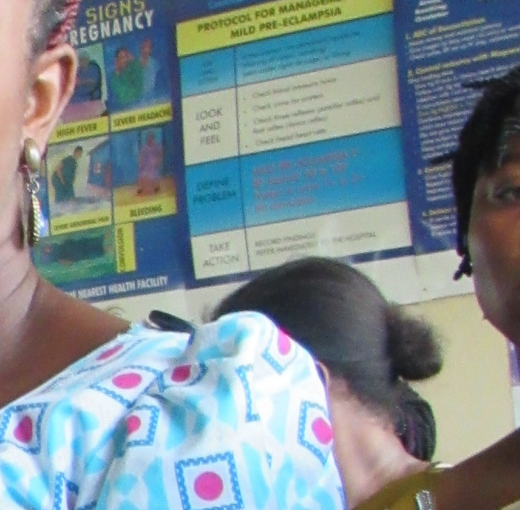

○)

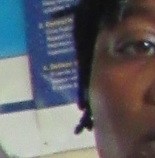




\section{BACKGROUND}

Despite global efforts to reduce preventable maternal and neonatal mortality, Nigeria's maternal mortality ratio is estimated at 576 deaths per 100,000 live births and neonatal death is estimated at 37 per 1,000 live births .

Maternal and newborn deaths due to pre-eclampsia and eclampsia (PE/E) are preventable, yet in Nigeria this is the most significant direct cause of maternal mortality.

To appreciate the enormity of this problem at country and state levels, Population Council conducted a landscape analysis on PE/E in seven states in Nigeria were to understand Nigeria's PE/E context, including assessment of evidence gaps over the last 15 years, and to determine priority areas for research and interventions around PE/E. This brief presents study findings from Ebonyi State on post-intervention landscape changes in:

- Programmatic and policy support for PE/E prevention and treatment;

- PHC facilities' capacity to manage PE/E;

- Provider knowledge and competence in preventing, detecting, and managing $\mathrm{PE} / \mathrm{E}$; and

- Community awareness, beliefs, and experiences around PE/E.

\section{METHODS}

This PE/E study is a pre/post, cross-sectional design with qualitative and quantitative data collected at baseline (2015) and endline (2018). Data collection activities include in-depth interviews (IDIs) with policy makers, survivors of PE/E and laboratory professionals; focus group discussions (FDGs) with men and women from the community; and surveys and observations to assess provider capacity in antenatal care and facility readiness and capacity to identify, treat, and manage PE/E at the primary and secondary levels.

Table 1 describes data collection activities at baseline and endline in Ebonyi State.

\section{Table 1: Data collection activities in Ebonyi}

\begin{tabular}{|l|c|c|}
\hline \multirow{2}{*}{} & \multicolumn{2}{|c|}{$\mathrm{n}$} \\
\cline { 2 - 3 } & Baseline & Endline \\
\hline IDIs with policy makers & 10 & 18 \\
IDIs with PE/E survivors & 5 & 6 \\
IDIs with laboratory professionals & 5 & 6 \\
FGDs with married men \& women & 4 & 4 \\
Surveys with providers & 6 & 89 \\
Assessments of health facilities & 20 & 57 \\
Observations of ANC visits & 10 & 119 \\
\hline
\end{tabular}

PRE-ECLAMPSIA/ECLAMPSIA (PE/E)

IN BRIEF

- $\quad P E$ is a condition in pregnant women marked by an increase in blood pressure and protein in urine after 20 weeks gestation; eclampsia is a lifethreatening condition characterized by convulsions in women with PE.

- $\quad$ Providing high quality ANC improves the prevention and early detection of $\mathrm{PE}$ and can prevent its progression to eclampsia.

- $\quad \mathrm{PE} / \mathrm{E}$ can be managed by administering anti-hypertensive drugs and $\mathrm{MgSO}_{4}$.

\section{CORE INTERVENTION}

- Intervention research occurred in Cross River, Ebonyi, and Kogi states, in 2016 and 2017, and follow-up data was collected from May-June 2018.

- 557 primary health care (PHC) providers received one month of training with continuous mentorship to identify hypertension and $\mathrm{PE} / \mathrm{E}$, treat with $\mathrm{MgSO}_{4}$, and refer to secondary facilities.

- The primary objective was to facilitate task-shifting to $\mathrm{PHC}$ providers of $\mathrm{MgSO}_{4}$ loading dose administration and secondary facility referral for improved PE/E detection, treatment, and management.

\section{ADDITIONAL ANTIHYPERTENSIVE}

\section{INTERVENTION}

- 43 community health extension workers (CHEWs) in PHCs were trained to measure and categorize hypertension in pregnancy and to manage using alpha methyldopa.

- 29 comparison group providers did not prescribe antihypertensives but referred women with hypertension to secondary facilities.

- Provider capacity was evaluated by following pregnancy outcomes of 54 hypertensive women attending ANC at intervention and comparison facilities from November 2016 to Dec. 2017.

The Ending Eclampsia project seeks to expand acces to proven, underutilized interventions and commodities for the prevention, early detection, and treatment of pre-eclampsia and eclampsia and strengthen global partnerships. 


\section{POLICY INFLUENCE}

Researchers interviewed six policy makers from the local and state levels, including local government area (LGA) health facility managers to understand their knowledge of programs and policies to detect, prevent, and manage $\mathrm{PE} / \mathrm{E}$ in in Ebonyi. Policymakers were asked about efforts in their LGA or state to advance national PE/E policies as well as to assess their support for task shifting to $\mathrm{PHC}$ providers for PE/E management in accordance with Nigerian national protocol. They were asked about the accessibility, affordability, and utilization of antihypertensive drugs and magnesium sulfate $\left(\mathrm{MgSO}_{4}\right)$ in their communities.

Policy makers emphasize the need for early antenatal care (ANC) registration. There is also a concern that many women in Ebonyi State continue to prefer home deliveries, despite efforts to encourage facility deliveries. Policy makers recognize the structural barriers to ANC that women face when accessing health facilities, including poor infrastructure and travel costs. To overcome these barriers, policymakers support the idea of task shifting to PHC workers for PE/E management - a major step forward from baseline.

\section{"At the facility level, the trainings that these health workers received have really empowered them; their capacity has been built... in 2015 many of them did not know what magnesium sulphate was but now in all the health facilities, the majority of the people trained can manage pre-eclampsia. They notice the warning signs; they give the loading dose and then refer."}

- State policy maker, 2018
Challenges of insufficient manpower at the PHC level remain, and policy makers acknowledge that women often bypass primary facilities knowing that providers are more likely to be available at the secondary level. There is some discrepancy between policy makers' and community members' perceptions of drug costs for treating PE/E and the relative barrier those costs present to pregnant women. Policy makers report that ANC drugs are often free and accessible, community members perceive drug and hospital costs to be one barrier to accessing ANC.

\section{FACILITY CAPACITY \& PREPAREDNESS}

At baseline, researchers assessed 20 health facilities in Ebonyi and found that less than 25\% were fully equipped to detect, manage, and treat PE/E. At endline, 57 primary and secondary facilities were assessed, and $86 \%$ of facilities now have guidelines for managing PE/E compared to $15 \%$ at baseline $(p<0.001)$.

At baseline, 10 facilities (50\%) had all necessary ANC equipment to detect PE/E; at endline, 45 facilities (79\%) had all equipment. While at baseline, $30 \%$ of facilities at least sometimes used $\mathrm{MgSO}_{4}$ to manage $\mathrm{PE} / \mathrm{E}$, at endline, $86 \%$ of facilities reported always or sometimes using $\mathrm{MgSO}_{4}$.

Through direct observation, researchers identified the number of facilities that stocked key equipment to detect and manage $\mathrm{PE} / \mathrm{E}$ and treat $\mathrm{MgSO}_{4}$ toxicity. Improvements were observed for stocking all key equipment except urine catheters, though the overall number of facilities with urine catheters increased 5\% (Figure 1).

At baseline, only three facilities in Ebonyi State reported always using $\mathrm{MgSO}_{4}$ to treat $\mathrm{PE} / \mathrm{E}$ whereas, at endline,

Figure 1: Facilities with key equipment to detect and manage $\mathrm{PE} / \mathrm{E}$ and $\mathrm{MgSO}_{4}$ toxicity (\%)

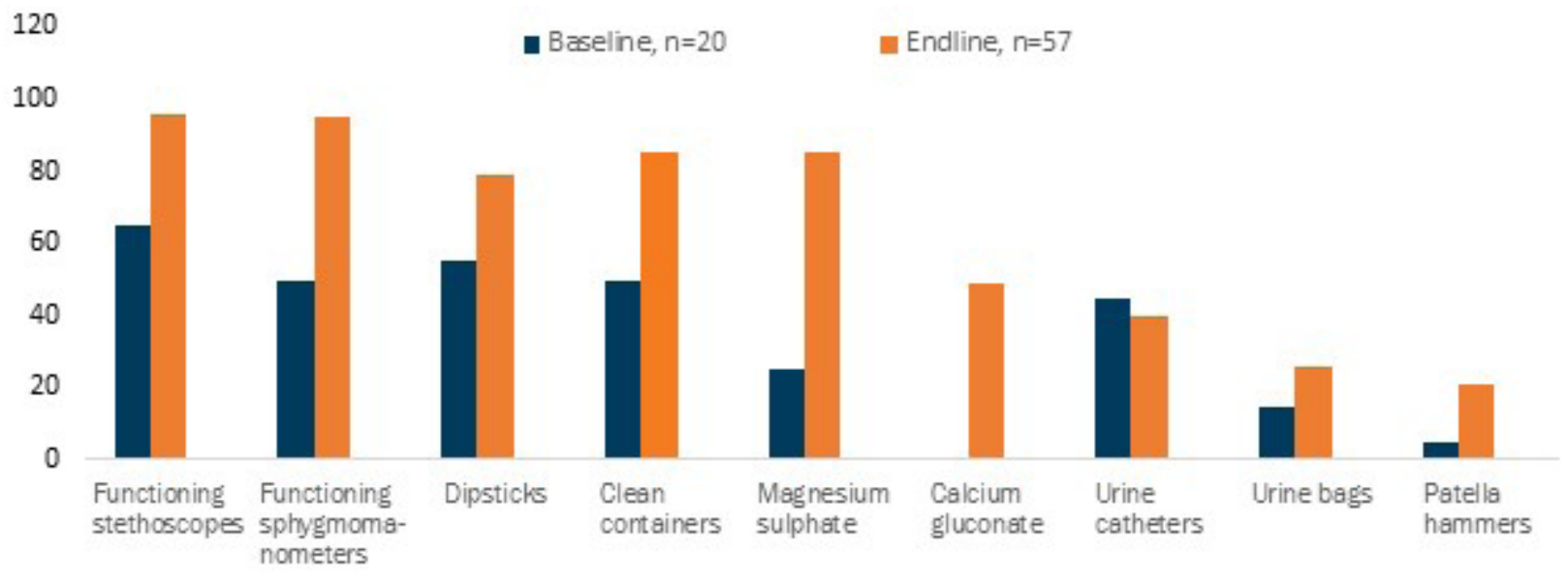


27 facilities always used $\mathrm{MgSO}_{4}$. Though not statistically significant, more facility managers at endline reported sometimes using $\mathrm{MgSO}_{4}$ (22 vs. three at baseline) and significantly fewer managers at endline said $\mathrm{MgSO}_{4}$ is never used in their facilities compared to baseline $(n=8$ vs. $14 ; p<0.001$ ). These findings suggest that, while gaps persist, more facilities are using $\mathrm{MgSO}_{4}$ for patients when necessary.

At baseline, only $33 \%$ of the facilities that stocked $\mathrm{MgSO}_{4}$ received it as part of their regular supply from central medical stores, but at endline $93 \%$ of facilities reported this procurement method. At endline, no facilities in Ebonyi State reported that clients must purchase drugs from the market themselves.

A critical component of managing and treating severe $\mathrm{PE} / \mathrm{E}$ is the capacity to conduct emergency obstetric and neonatal care (EmONC) by providing key signal functions and essential drugs to women throughout the pregnancy continuum (pregnancy, labor and delivery, and postnatal periods). Of all facilities observed in Ebonyi at endline, four (7\%) stock all essential equipment, and 36 (63\%) stock at least half of all essential supplies. Table 2 compares facilities' capacity to provide EmONC signal functions at baseline and endline.

\begin{tabular}{|l|c|c|c|c|}
\hline \multirow{2}{*}{ Table 2: Signal functions for EmONC } \\
\hline & \multicolumn{3}{|c|}{ Baseline, $\mathrm{n}=20$} & \multicolumn{2}{c|}{ Endline, $\mathrm{n}=57$} \\
\cline { 2 - 5 } & Capacity & $\begin{array}{c}\text { Done } \\
\text { in past } \\
3 \text { mos }\end{array}$ & Capacity & $\begin{array}{c}\text { Done } \\
\text { in past } \\
3 \text { mos }\end{array}$ \\
\hline $\begin{array}{l}\text { Perenteral } \\
\text { antibiotics }\end{array}$ & 17 & 13 & 51 & 36 \\
$\begin{array}{l}\text { Parenteral oxytocics } \\
\begin{array}{l}\text { Parenteral } \\
\text { anticonvulsants } \\
\text { (Mgso })\end{array}\end{array}$ & 18 & 16 & 52 & 43 \\
$\begin{array}{l}\text { Manual placenta } \\
\text { removal }\end{array}$ & 13 & 8 & 45 & 24 \\
$\begin{array}{l}\text { Retained products } \\
\text { removal }\end{array}$ & 13 & 11 & 39 & 18 \\
$\begin{array}{l}\text { Assisted vaginal } \\
\text { delivery }\end{array}$ & 13 & 10 & 17 & 11 \\
$\begin{array}{l}\text { Blood transfusion } \\
\text { Caesarean section }\end{array}$ & 7 & 4 & 18 & 3 \\
$\begin{array}{l}\text { Neonatal } \\
\text { rescuscitation }\end{array}$ & 11 & 8 & 44 & 24 \\
\hline
\end{tabular}

\section{PROVIDER KNOWLEDGE \& SKILLS}

Eighty-nine providers from primary and secondary facilities in Ebonyi responded to questions about preventing, detecting, and treating PE/E. At endline, more providers could correctly identify pre-eclampsia than at baseline (86\% compared to $78 \%$ ).
Compared to baseline, providers can now better recognize and manage hypertension in pregnancy. While not statistically significant, $90 \%$ of providers correctly diagnosed hypertension at endline compared to $84 \%$ at baseline, and significantly more providers use antihypertensive drugs to manage hypertension ( $28 \%$ compared to $84 \% ; p<0.001$ ). These improvements are encouraging - in Ebonyi State, providers received additional training on identification and management of hypertension in pregnancy.

Figure 2: Providers' knowledge of drugs for PE/E prevention and management (\%)

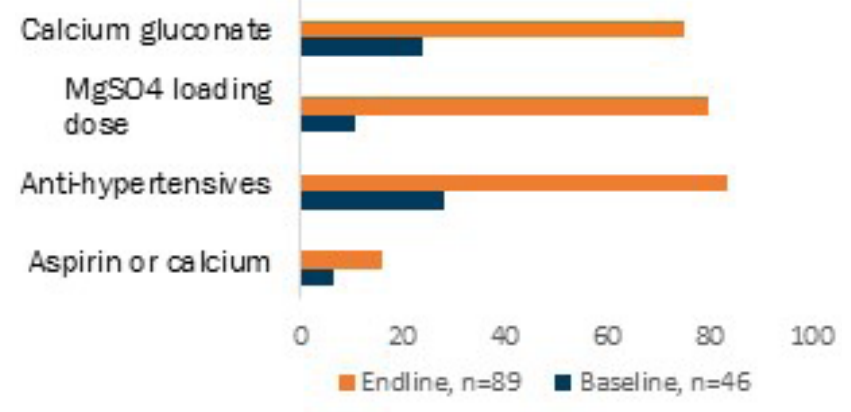

A key component of task shifting from secondary to primary facilities is PHC providers' capacity to prevent and manage $\mathrm{PE} / \mathrm{E}$ using antihypertensive drugs, $\mathrm{MgSO}_{4}$, and calcium gluconate to treat $\mathrm{MgSO}_{4}$ toxicity. Figure 2 highlights improvements in drug knowledge among PHC providers from baseline to endline.

This intervention is based on a modified Pritchard method for administering intramuscular loading and maintenance doses of $\mathrm{MgSO}_{4}$ to treat severe $\mathrm{PE}$ and prevent the onset of eclampsia. At baseline, only $11 \%$ and $2 \%$ of providers could correctly describe the loading and maintenance doses of $\mathrm{MgSO}_{4}$, respectively. At endline, $80 \%$ of providers know the appropriate loading dose and $55 \%$ know the correct maintenance dose $(p<0.001$ and $p<0.001$, respectively). Similarly, while at baseline approximately one-quarter of providers knew the correct antidote to $\mathrm{MgSO}_{4}$ toxicity, more than three-quarters of providers now recognize calcium gluconate as the correct antidote $(p<0.001)$.

\section{QUALITY OF CARE}

Quality of care was assessed at endline through 119 observations of client-provider interactions during ANC visits, as well as qualitative data generated through four men's and women's community focus groups, and six interviews with $\mathrm{PE} / \mathrm{E}$ survivors. Figure 3 compares the proportions of ANC components that clients received at baseline and endline. Improvements in the provision 


$$
\text { - Baseline, } \mathrm{n}=10 \quad \text { Endline, } \mathrm{n}=119
$$

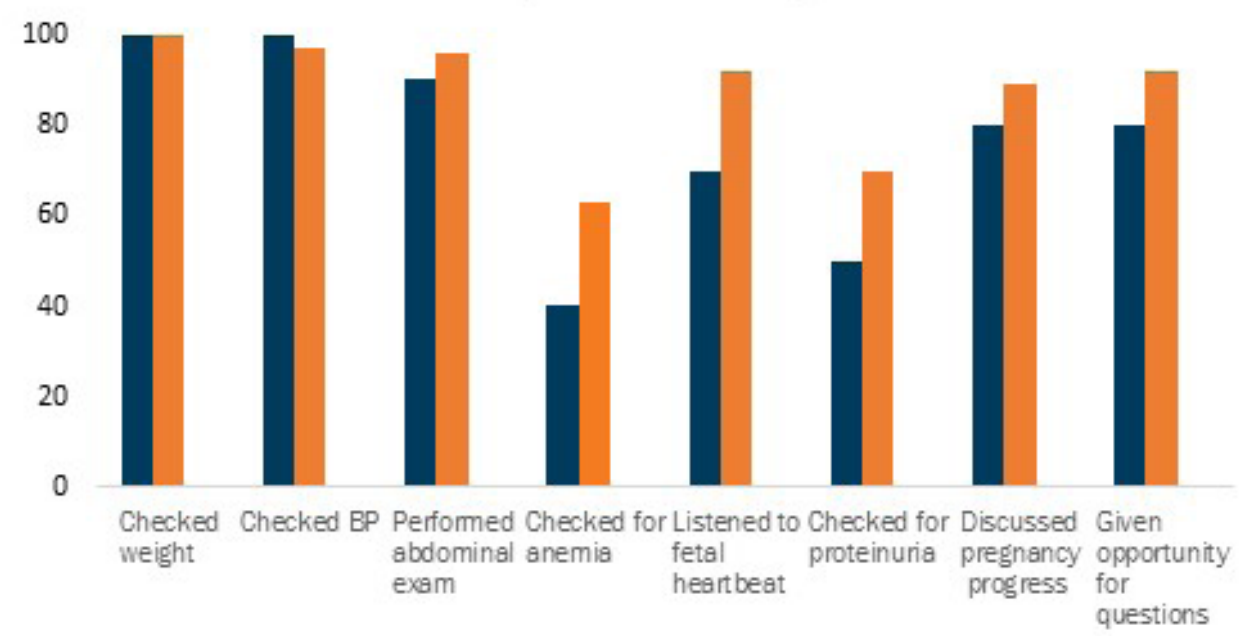

of all eight essential ANC components were observed, except in the number of observations of blood pressure (BP) checks which fell slightly.

Client reports confirm Figure 3 findings that BP, proteinuria, and weight screenings regularly occur during ANC. However, $30 \%$ of women still do not receive regular proteinuria checks, a critical part of $\mathrm{PE} / \mathrm{E}$ detection. Consequently, once high BP is detected, some women are referred to secondary facilities where they receive a loading dose of $\mathrm{MgSO}_{4}$. Failure to conduct urinalysis may be due to supply chain gaps, and there are mixed perspectives about supply procurement among laboratory staff. Some lab staff report that regular, monthly procurements to their facilities prevent critical supply stock outs, but others emphasize the need for greater external support for adequate resource supply.

Although $92 \%$ of women are given the opportunity to ask questions during ANC, most women in Ebonyi State report not discussing PE/E explicitly with their providers. Lack of discussion may be due to women's lack of information or social norms regarding information sharing with women. Only $15 \%$ of providers were observed offering education about potential pregnancy complications and delivery options. Nearly all survivors of PE/E report hearing about maternal or fetal death as a potential complication of $\mathrm{PE} / \mathrm{E}$, but knowledge gaps persist on warning signs of and prevention strategies for hypertension.

\footnotetext{
"Based on what they saw in my system, they called the doctor, the doctor told them to send me to him. I don't know what they saw, I even asked them, 'What did you see'? they said that the doctor will tell me. When I went there, the doctor did not tell me anything, maybe they told my husband. They cannot say everything
}

to a pregnant woman so that she will not be thinking. If they told my husband, I do not know; I kept asking my husband, but he did not tell me anything, he did not tell me what is the problem so that if it is something that can be prevented, let me do my best to prevent it. Even if it is from my food intake, he refused to talk, he said that "they said that they saw something'."

- Pre-eclampsia survivor, 2018

Compared to other states, multiple survivors of $\mathrm{PE} / \mathrm{E}$ in Ebonyi State report natural deliveries, rather than caesarean section (CS). This is encouraging considering $\mathrm{PHC}$ providers in Ebonyi received supplemental training on the use of anti-hypertensive drugs to prevent $\mathrm{PE}$ progression to severe $\mathrm{PE} / \mathrm{E}$; it is possible that women with PE were managed and were able to safely undergo vaginal births rather than emergency CS. Women report being well taken care of while in the hospital and during delivery.

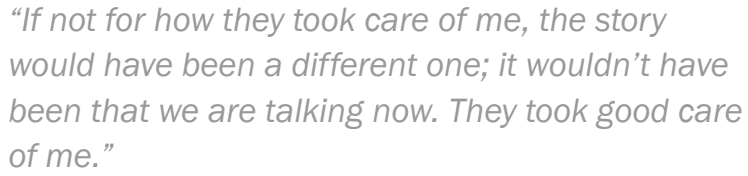

- Pre-eclampsia survivor, 2018

\section{COMMUNITY KNOWLEDGE \& PERCEPTIONS}

Women in Ebonyi State seek ANC services, though they often wait until the sixth or seventh month of their pregnancy. At baseline, financial concerns from men and women continue to influence women's health-seeking behaviors. FGD participants said that hospital bills and associated drug costs, rather than transportation costs, 
prevent women from seeking ANC services. However, $\mathrm{PE} / \mathrm{E}$ survivors report that cost was not a factor in their decision to seek care; instead, they and their husbands were concerned with seeking expert care. Some women discuss traditions of home births as a secondary barrier to health services, though traditional birth practices came up during FGDs less frequently than at baseline.

Mistrust between community members and the health system seems to be less prevalent than at baseline, and community members discuss the importance of ANC registration and seeking health services in a facility rather than from traditional healers or religious leaders. Traditional beliefs about the causes of hypertension, including witchcraft and over-thinking, persist but appear less pervasive than at baseline.

\section{SURVIVOR'S EXPERIENCES}

While hospital utilization is high among PE/E survivors in Ebonyi State, at least one woman stated that, despite receiving a hospital referral following her ANC visit, she chose to go to church to pray for her pregnancy and ultimately delivered her baby at home, citing faith in God as the primary reason for not adhering to her referral. Compared to changes seen in Cross River and Kogi states, religious influence remains high within the health system in Ebonyi. Given its consistencies to baseline, this finding suggests that religious leaders should be better integrated into the health system as sources of information and referral partners.

\section{"Each time I go for ANC they do prayers then they will check BP, weight, and the baby."}

\section{- Pre-eclampsia survivor, 2018}

Women in Ebonyi attended multiple ANC visits, though at least one woman reported registering for ANC because she experienced uncomfortable symptoms, rather than for standard, pregnancy-related check-ups. Unlike at baseline, survivors at endline report that both their BP and urine were checked during ANC visits. Many survivors received information about possible health complications associated with high BP, such as maternal and fetal death, though, similar to baseline, less information was given about the implications of proteinuria.

\section{DISCUSSION}

Policy maker views show support for scaling up taskshifting of antihypertensive drugs and $\mathrm{MgSO}_{4}$ loading dose administration and referral to PHC providers. Policy makers differ from community members and survivors in their perceptions of cost as a barrier to facility- based care for hypertension. While policymakers state that antihypertensive drugs are free and accessible, community members describe drug costs and hospital bills as key perceived barriers to women seeking maternal health care at PHCs or hospitals. Contrastingly, survivors of PE/E state that cost did not factor into their decisions to seek care. Further exploration of differences between perceived and actual cost barriers is warranted.

Facility capacity to detect, treat, and manage PE/E has improved, and more facilities are able to procure and use $\mathrm{MgSO}_{4}$ to treat patients. Supported by greater facility resource capacity and training, provider capacity to identify and manage hypertension and PE also improved. Inconsistencies remain in the number of women receiving BP and proteinuria checks before receiving secondary referrals, with many women being referred immediately after detection of high BP rather than after high BP and proteinuria are detected. Supply stock outs of urinalysis kits, rather than provider training, are likely the cause rather than insufficient provider knowledge. Compared to other states where community components were stronger, women's awareness and ability to communicate with $\mathrm{PHC}$ providers during ANC remains an area for improvement.

One limitation of the data is the unequal sample sizes from ANC observations at baseline and endline. However, triangulating across different data sources and types renders our study findings robust.

\section{RECOMMENDATIONS}

- $\quad$ Streamline state procurement and enhance linkages to central distribution system;

- Continue mentoring efforts and supportive supervision for PHC providers to detect and manage hypertension in pregnancy, including PE/E;

- Engage religious leaders to better promote healthseeking behaviors; and

- Increase community engagement through women's groups and other public fora to promote health education on hypertensive disorders of pregnancy.

\section{REFERENCES}

1. DHS Nigeria, 2013. 